\title{
Effect of Pre-harvest Application of Ca, K, B and Zn on Yield and Quality of Mango (Mangifera indica L.) cv. Langra
}

\author{
Anugya Kumari*, Rewati Raman Singh and Manoj Kundu \\ Department of Fruit and Fruit Technology, Bihar Agricultural University, \\ Sabour-813210, Bhagalpur, Bihar, India \\ *Corresponding author
}

\section{A B S T R A C T}

The present study was conducted to investigate the role of calcium, potassium, boron and zinc on yield and quality of mango cv. Langra. The

\section{Keywords}

Mango, Potassium, Calcium, Boron, Zinc, Yield, Quality

Article Info

Accepted:

18 May 2020

Available Online:

10 June 2020 treatments includes $\mathrm{CaCl}_{2} 4 \%, \mathrm{CaCl}_{2} 6 \%, \mathrm{CaCl}_{2} 8 \%, \mathrm{~K}_{2} \mathrm{SO}_{4} \quad 1.0 \%$, $\mathrm{K}_{2} \mathrm{SO}_{4} 1.5 \%, \mathrm{~K}_{2} \mathrm{SO}_{4} 2.0 \%$, Borax 1.0\%, Borax 1.5\%), Borax 2.0\%, $\mathrm{ZnSO}_{4}$ $0.2 \%, \mathrm{ZnSO}_{4} 0.4 \%, \mathrm{ZnSO}_{4} 0.8 \%$ ) and $\mathrm{T}_{13}$ control. Spraying was done 30 days before anticipated day of harvesting. The result revealed that among all the treatments borax $2 \%$ was most effective in increasing fruit weight, pulp weight, and pulp percentage. Whereas $\mathrm{CaCl}_{2} 4.0 \%$ had given highestyield/plant with better fruit quality attributes. On the other hand number of fruits per plant and fruit quality parameters such as TSS, TSS: acid ratio, sugars, total carotenoids, ascorbic acid content, total phenolics was highest in borax $1 \%$. Thus, present study concluded that borax $1 \%$ and $\mathrm{CaCl}_{2} 4 \%$ was most efficient in enhancing yield and yield attributes as well as in retaining the quality of mango fruits.

\section{Introduction}

Mango (Mangifera indica L.) is choicest fruit crop of India, cultivated in tropical and subtropical region around the world. India is leading producer and around $2.516 \mathrm{MT} / \mathrm{ha}$ area is under mango cultivation with an annual production of $18431 \mathrm{MT}$. Although, production of this crop is very high but the productivity is quite lower than other fruit crops (Anonymous, 2015). Mango fruits are esteemed due its pleasing aroma, marvellous flavour, luscious taste, attractive colour with high nutritive value, which has fascinated the market.

Among the various cultivars of northern India, Langrais one of the main cultivar which is widely cultivated and preferred by the consumers. Although it is one of the choicest 
variety but its yield is very low. This main reason for lower yield is its biennial bearing habit, low fruit set, excessive fruit drop, poor fruit growth, development of abscission layer and deficiency of nutrients in different part of the country.

Application of different macro and micronutrients has shown immense potential to increase the yield and productivity of mango with improved fruit quality (Selvaraj et al., 2000). Potassium plays major role in many biochemical and physiological processes such as growth, yield, quality and biotic and abiotic tolerance (Cakmak, 2005; Pettigrew, 2008). The potassium treatments improve the productivity of several mango cultivars (Baiea et al., 2015; Taha et al., 2014). On the other hand, calcium spraying increased the productivity of mango due to the reduction of abscission layer formation (Kumar et al., 2006). Calcium application safely supplements endogenous calcium to fresh fruits during development of fruits (Raese and Drake, 2000). Boron improves translocation of sugar, cell wall synthesis and their structure as well as lignifications that affect fruit quality (Blevins and Lukaszewski, 1998). Zinc plays an important role in enzymatic reactions and helps in better growth and development. It also regulates the metabolism of protein as well as carbohydrate and improves the auxin content which helps in fruit retention.

Various experiments have been conducted on foliar spray of micro-nutrients, shown significant response to improve yield and quality of fruits and found 10 to 20 times more efficient than soil application (Shukla, 2011; Zaman and Schumann, 2006).

Present study was planned to evaluate the response of foliar spray of calcium, potassium, boron and zinc on yield and quality of mango fruits cv. Langra.

\section{Materials and Methods}

\section{Experimental site}

The research was done in mango orchard of Bihar Agricultural College, Sabour, Bhagalpur.

\section{Plant materials and treatments}

For this study 21 years old mango trees (Mangifera indica L.) cv. Langra of uniform vigour, growing in a compact block were selected. All the trees were managed under similar cultural schedule during whole period of study. Randomized block design (RBD) with 13 treatments and 3 replications for each, were used for this experiment.

The details of experimental treatment plan employed in this study are as follow: $\mathrm{T}_{1}\left(\mathrm{CaCl}_{2} 4 \%\right), \mathrm{T}_{2}\left(\mathrm{CaCl}_{2} 6 \%\right), \mathrm{T}_{3}\left(\mathrm{CaCl}_{2} 8 \%\right)$, $\mathrm{T}_{4} \quad\left(\mathrm{~K}_{2} \mathrm{SO}_{4} 1.0 \%\right), \quad \mathrm{T}_{5} \quad\left(\mathrm{~K}_{2} \mathrm{SO}_{4} 1.5 \%\right), \quad \mathrm{T}_{6}$ $\left(\mathrm{K}_{2} \mathrm{SO}_{4} 2.0 \%\right), \mathrm{T}_{7}$ (Borax 1.0\%), $\mathrm{T}_{8}$ (Borax $1.5 \%), \mathrm{T}_{9}($ Borax $2.0 \%), \mathrm{T}_{10}\left(\mathrm{ZnSO}_{4} 0.2 \%\right)$, $\mathrm{T}_{11}\left(\mathrm{ZnSO}_{4} 0.4 \%\right), \mathrm{T}_{12}\left(\mathrm{ZnSO}_{4} 0.8 \%\right)$ and $\mathrm{T}_{13}$ Control (Water spray). These nutrients were applied as foliar spray at 30 days before harvest of fruits.

\section{Observation recorded}

\section{Yield attributing characters and yield}

Total numbers of fruits for each replication under each treatment were counted manually. Fruit weight, Pulp weight, peel and stone weight, yield per plant as well as Yield per ha were recorded. Fruit length and width was measured by using Vernier calliper (mm). Specific gravity and fruit volume was calculated by conventional water displacement method. Pulp: stone ratio was calculated by dividing weight of pulp with weight of stone and also edible: non-edible ratio was estimated. 


\section{Fruit quality attributes}

Total soluble solids were recorded with the help of hand refractometer. Titratable acidity was calculated by standard titration method (AOAC, 2000). TSS: TA ratio was calculated by dividing the TSS with titratable acidity. Total sugar was done by using Lane and Eynone (1923) method and expressed in percentage. Total carotenoids was evaluated by the protocol of Roy (1973) and values were expressed as mg $100 \mathrm{~g}^{-1}$ fresh weight basis (FW).Ascorbic acid was estimated by 2 , 6-dichlorophenol indophenol dye method (Jones and Hughes, 1983) and results were expressed in mg 100/g FW. Total phenols were extracted by a method described by Singleton et al., (1999) and were expressed as microgram of gallic acid equivalent/100 g FW (mg GAE g ${ }^{-1} \mathrm{FW}$ ). Total Antioxidant activity was calculated according to the protocol of Apak et al., (2004) CUPRAC assay and expressed as $\mu \mathrm{mol}$ Trolox equivalent/100 gFW.

\section{Statistical analysis}

The Data were analyzed using statistical analysis software SAS 9.2 and means were calculated by using Duncan's multiple range test. Mean difference were tested by ' $F$ ' test at five per cent level of significance. Treatments were compared at five per cent level of significance.

\section{Results and Discussion}

\section{Yield attributing characters}

Fruit weight, number of fruits per plant, yield per plant and yield per ha

Total number of fruits from each plant, fruit weight, yield/plant and yield/ha were presented in table-1.Result revealed that all the treatments have significantly higher number of fruits per plant. Borax $1 \%$ (191.33) had maximum number of fruits per plant followed by borax $1.5 \%$ (184.33) while minimum (140.00) in untreated plant. Fruit weight was highest in borax 2.0\% (353.07 gm) followed by $\mathrm{CaCl}_{2} 4 \%$ (351.83) and minimum in $\mathrm{K}_{2} \mathrm{SO}_{4} 1.5 \%$ (275.73 gm). Foliar application of boron showed positive effect because it promotes cell division, expansion, sugar metabolism and accumulation of carbohydrates (Sourour, 2000). It also helps in better photosynthesis, accumulation of starch and auxin synthesis. The balance auxin in plant regulates the fruit drop and helps in maximum number of fruit retention. Similar results were reported in mango, papaya and guava (Sankar et al., 2013; Kavitha, 2000; Sarolia et al., 2007). The appreciable improvement in fruit weight by boron application had been reported by Pathak et al., 2011, Bhatt et al., 2012; Bhowmick et al., 2012; Singh et al., 2012; Yadav et al., 2013. It might be due to involvement of boron in hormonal metabolism, cell division and expansion and also enhanced assimilates accumulation from other parts to developing fruits However, the maximum yield/plant and yield/ha (63.79 kg, $6.37 \mathrm{t} / \mathrm{ha}$ respectively) was recorded in $\mathrm{CaCl}_{2} 4.0 \%$ while the minimum in control. Boron and calcium significantly increased yield, it might be an increase in fruit number as fruit drop reduced, higher fruit weight. These results were in agreement with earlier studies of Singh and Maurya, 2004 and Singh et al., 2012 in mango.

Fruit weight, fruit length, width, volume and specific gravity

Table.2 showed that treatments have significant effects on fruit weight, length width and volume. The maximum fruit length and volume was recorded in $\mathrm{CaCl}_{2} 4.0 \%$. Appreciable improvement in fruit length and volume by $\mathrm{CaCl}_{2}$ application might be because of hormonal metabolism, increased cell 
division and expansion, synthesis and changes of carbohydrates and carbohydrate enzyme (Rani and Brahmachari, 2001; Banis et al., 1997). The similar reports were found by Kulkarni and Yewale (2012) and Karemera and Habimana (2014). The maximum fruit width $(80.81 \mathrm{~mm})$ was recorded in $\mathrm{ZnSO}_{4}$ $0.8 \%$ while minimum in control $(69.287 \mathrm{~mm})$. This increase in width might be due to direct role of zinc in hastening the process of cell division and cell elongation. Positive effect of zinc on fruit width was also reported by Singh et al., (2013). However, specific gravity was recorded highest in borax $1.5 \%$ followed by borax $2 \%$ while minimum in $\mathrm{K}_{2} \mathrm{SO}_{4} 1.0 \%$.

\section{Pulp and stone characteristics and Edible: Non-edible ratio}

The maximum pulp weight was recorded in borax 2\% (290.60 gm) followed by $\mathrm{CaCl}_{2} 4 \%$ while the minimum pulp weight was found in control (213.66 gm). Boron increased fruit weight by more accumulation of photosynthates in the matured fruits. Result was in close conformity with Bhowmick et al., 2012; Karemera et al., 2014 and Bhusan and Panda, 2015. Whereas, maximum peel weight was recorded in control (37.13 gm) followed by $\mathrm{K}_{2} \mathrm{SO}_{4} 1.5 \%$ (38.55 gm) while minimum in $\mathrm{CaCl}_{2} 4.0 \%$ (23.42 gm). The maximum stone weight was recorded in $\mathrm{CaCl}_{2} 4.0 \%$ followed by $\mathrm{CaCl}_{2} 8 \%$ while the minimum stone weight was found in control (Table-3).

From table-4it was clear that maximum pulp percentage was recorded in borax $2 \%$ followed by $\mathrm{CaCl}_{2} 4 \%$ and the minimum in $\mathrm{K}_{2} \mathrm{SO}_{4} 1.5 \%$. Highest peel percentage was recorded in control (13.47\%) followed by $\mathrm{K}_{2} \mathrm{SO}_{4} 1.5 \%$ (13.26\%) while minimum peel percentage was found in $\mathrm{CaCl}_{2} 4 \%(6.66 \%)$.

These finding of Bhusan et al., (2015) and Bhowmick et al., (2012) were showed similar result. The stone percentage was recorded highest in $\mathrm{CaCl}_{2} 8 \%$ and minimum in $\mathrm{K}_{2} \mathrm{SO}_{4} 1 \%$ (Table-4). Fruit pulp: stone ratio was recorded highest in $\mathrm{K}_{2} \mathrm{SO}_{4} 1 \%$ followed by borax $2 \%$ while it was minimum in borax $1.5 \%$. The maximum edible: non-edible ratio was recorded in borax $2 \%$ followed by $\mathrm{CaCl}_{2}$ $4.0 \%$ (Table-3). Similar findings were observed by Karemeraa and Habimana, 2014).

\section{Fruit quality attributes}

\section{Total soluble solids}

The maximum TSS $\left(21.08^{\circ} \mathrm{B}\right)$ was observed in borax $1 \%$ followed $\mathrm{CaCl}_{2} 4 \%$ and minimum in control fruits (Table-5).

Increase in TSS by boron application attributed to the rapid mobilization of sugars and other soluble solids from leaves to developing fruits. These findings were in agreement with the findings of Bhowmick et al., (2012), Singh et al., (2012), Nehete et al., (2011) and Bhatt et al., (2012).

\section{Titratable acidity (\%)}

A critical review of data (Table-5) indicated that the highest titratable acidity $(0.29 \%)$ was found in untreated fruits whereas it was lowest $(0.24 \%)$ in $\mathrm{ZnSO}_{4} 0.8 \%$ as followed $\mathrm{ZnSO}_{4} 0.2 \%$. $\mathrm{ZnSO}_{4}$ effectively reduced acidity by accumulation of more total soluble solids. Enzymatic reactions need $\mathrm{Zn}$ for transformation of carbohydrates, hexokinase activity and sugar conversion (Dutta and Dhua, 2002). Similar effect of zinc was found in other fruit crops such as aonla, mango etc. (Meena et al., 2014; Panday and Jain, 2014).

\section{TSS: TA ratio}

Foliar application of borax $1.0 \%$ had significantly highest TSS: TA ratio as compare to other treatments (table-5). 
Table.1 Effect of chemicals on yield attributing characters of mango cv. Langra

\begin{tabular}{|c|c|c|c|c|}
\hline Treatments & $\begin{array}{l}\text { Total number } \\
\text { of fruits/plant }\end{array}$ & $\begin{array}{l}\text { Fruit weight } \\
\text { (gm) }\end{array}$ & $\begin{array}{c}\text { Yield /plant } \\
\text { (kg) }\end{array}$ & $\begin{array}{c}\text { Yield/ha } \\
(\mathbf{t})\end{array}$ \\
\hline $\mathrm{CaCl}_{2}(4.0 \%)$ & $181.33^{b c}$ & $351.83^{\mathrm{ab}}$ & $63.79^{a}$ & $6.37^{\mathrm{a}}$ \\
\hline $\mathrm{CaCl}_{2}(6.0 \%)$ & $178.33^{\mathrm{cde}}$ & $339.60^{b c}$ & $60.56^{\mathrm{ab}}$ & $6.05^{\mathrm{ab}}$ \\
\hline $\mathrm{CaCl}_{2}(8.0 \%)$ & $176.00^{\text {def }}$ & $324.23^{\text {efg }}$ & $56.96^{\mathrm{bc}}$ & $5.69^{\mathrm{bc}}$ \\
\hline $\mathrm{K}_{2} \mathrm{SO}_{4}(1.0 \%)$ & $174.67^{\mathrm{ef}}$ & $334.83^{\text {cde }}$ & $58.47^{\mathrm{abc}}$ & $5.84^{\mathrm{abc}}$ \\
\hline $\mathrm{K}_{2} \mathrm{SO}_{4}(1.5 \%)$ & $168.00^{\mathrm{g}}$ & $275.73^{\mathrm{j}}$ & $46.30^{\mathrm{e}}$ & $4.63^{\mathrm{e}}$ \\
\hline $\mathrm{K}_{2} \mathrm{SO}_{4}(2 \%)$ & $165.67^{\mathrm{g}}$ & $295.36^{\mathrm{i}}$ & $48.94^{\mathrm{e}}$ & $4.89^{\mathrm{e}}$ \\
\hline Borax $(1.0 \%)$ & $191.33^{\mathrm{a}}$ & $312.36^{\text {fgh }}$ & $59.76^{\mathrm{abc}}$ & $5.97^{\mathrm{abc}}$ \\
\hline Borax (1.5\%) & $184.33^{b}$ & $325.28^{\mathrm{def}}$ & $59.96^{\mathrm{abc}}$ & $5.99^{\mathrm{abc}}$ \\
\hline Borax $(2.0 \%)$ & $173.67^{t}$ & $353.07^{\mathrm{a}}$ & $61.28^{\mathrm{ab}}$ & $6.12^{\mathrm{ab}}$ \\
\hline $\mathrm{ZnSO}_{4}(0.2 \%)$ & $164.33^{\mathrm{g}}$ & $307.66^{\mathrm{hi}}$ & $50.55^{\text {ed }}$ & $5.05^{\mathrm{ed}}$ \\
\hline $\mathrm{ZnSO}_{4}(0.4 \%)$ & $176.00^{\text {det }}$ & $311.33^{g h}$ & $54.81^{\mathrm{cd}}$ & $5.48^{\mathrm{cd}}$ \\
\hline $\mathrm{ZnSO}_{4}(0.8 \%)$ & $180.33^{\text {bcd }}$ & $338.00^{\mathrm{cd}}$ & $60.95^{\mathrm{ab}}$ & $6.09^{\mathrm{ab}}$ \\
\hline Control & $140.00^{\mathrm{h}}$ & $276.08^{j}$ & $38.65^{f}$ & $3.86^{\mathrm{f}}$ \\
\hline
\end{tabular}

Value indicates mean of three replicates. Different letters in the same column indicate significant differences at $\mathrm{P} \leq 0.05$ (Duncan's Multiple Range Test).

Table.2 Effect of chemicals on fruit growth and specific gravity of mango cv. Langra

\begin{tabular}{|c|c|c|c|c|}
\hline \multirow[t]{2}{*}{ Treatments } & \multicolumn{4}{|c|}{ Fruit growth } \\
\hline & $\begin{array}{c}\text { Fruit length } \\
(\mathbf{m m})\end{array}$ & $\begin{array}{l}\text { Fruit width } \\
\quad(\mathrm{mm})\end{array}$ & $\begin{array}{c}\text { Fruit volume } \\
\text { (cc) }\end{array}$ & $\begin{array}{c}\text { Specific } \\
\text { gravity }\end{array}$ \\
\hline $\mathrm{CaCl}_{2}(4.0 \%)$ & $101.30^{\mathrm{a}}$ & $78.28^{\mathrm{abc}}$ & $348.35^{\mathrm{a}}$ & $1.01^{\mathrm{a}}$ \\
\hline $\mathrm{CaCl}_{2}(6.0 \%)$ & $96.79^{\mathbf{a b}}$ & $77.15^{\text {abc }}$ & $336.28^{b c}$ & $1.01^{\mathrm{a}}$ \\
\hline $\mathrm{CaCl}_{2}(8.0 \%)$ & $95.85^{\mathbf{a b}}$ & $73.85^{\text {cde }}$ & $324.23^{\text {de }}$ & $1.00^{\mathrm{a}}$ \\
\hline $\mathrm{K}_{2} \mathrm{SO}_{4}(1.0 \%)$ & $95.65^{\mathrm{ab}}$ & $75.74^{\text {abcd }}$ & $341.66^{\mathbf{a b}}$ & $0.98^{\mathrm{a}}$ \\
\hline $\mathrm{K}_{2} \mathrm{SO}_{4}(1.5 \%)$ & $91.41^{\text {bc }}$ & $70.51^{\text {de }}$ & $275.73^{h}$ & $1.00^{\mathrm{a}}$ \\
\hline $\mathrm{K}_{2} \mathrm{SO}_{4}(2 \%)$ & $91.27^{\mathbf{b c}}$ & $70.49^{\text {de }}$ & $298.35^{\mathrm{g}}$ & $0.99^{\mathrm{a}}$ \\
\hline Borax $(1.0 \%)$ & $98.75^{\mathrm{a}}$ & $71.69^{\text {de }}$ & $309.34^{\mathbf{f}}$ & $1.01^{\mathrm{a}}$ \\
\hline Borax $(1.5 \%)$ & $100.23^{\mathrm{a}}$ & $75.08^{\text {bcd }}$ & $318.90^{\mathbf{e}}$ & $1.02^{\mathrm{a}}$ \\
\hline Borax $(2.0 \%)$ & $99.28^{\mathrm{a}}$ & $70.58^{\mathrm{de}}$ & $346.15^{\mathrm{a}}$ & $1.02^{\mathrm{a}}$ \\
\hline $\mathrm{ZnSO}_{4}(0.2 \%)$ & $98.96^{\mathrm{a}}$ & $78.08^{\mathrm{abc}}$ & $304.62^{\mathrm{gf}}$ & $1.01^{\mathrm{a}}$ \\
\hline $\mathrm{ZnSO}_{4}(0.4 \%)$ & $99.29^{\mathrm{a}}$ & $79.64^{\mathrm{ab}}$ & $305.23^{\mathrm{gf}}$ & $1.02^{\mathrm{a}}$ \\
\hline $\mathrm{ZnSO}_{4}(0.8 \%)$ & $100.12^{\mathrm{a}}$ & $80.81^{\mathrm{a}}$ & $331.37^{\mathrm{cd}}$ & $1.02^{\mathrm{a}}$ \\
\hline Control & $89.69^{c}$ & $69.28^{\mathrm{e}}$ & $273.35^{\mathrm{h}}$ & $1.01^{\mathrm{a}}$ \\
\hline
\end{tabular}

Value indicates mean of three replicates. Different letters in the same column indicate significant differences at $\mathrm{P} \leq 0.05$ (Duncan's Multiple Range Test) 
Table.3 Effect of chemicals on edible and non-edible portion of mango cv. Langra

\begin{tabular}{|c|c|c|c|c|c|}
\hline Treatments & $\begin{array}{c}\text { Pulp } \\
\text { weight } \\
\text { (gm) }\end{array}$ & $\begin{array}{l}\text { Peel weight } \\
\text { (gm) }\end{array}$ & $\begin{array}{c}\text { Stone } \\
\text { weight (gm) }\end{array}$ & $\begin{array}{l}\text { Edible: non- } \\
\text { edible ratio }\end{array}$ & $\begin{array}{l}\text { Pulp: } \\
\text { Stone } \\
\text { ratio }\end{array}$ \\
\hline $\mathrm{CaCl}_{2}(4.0 \%)$ & $289.40^{\mathrm{a}}$ & $23.42^{\mathrm{g}}$ & $39.01^{\mathrm{a}}$ & $4.63: 1^{a b}$ & $7.42: 1^{\text {cde }}$ \\
\hline $\mathrm{CaCl}_{2}(6.0 \%)$ & $277.18^{\mathrm{ab}}$ & $26.12^{\text {efg }}$ & $36.30^{\mathrm{abc}}$ & 4.44: $1^{\mathrm{abc}}$ & $7.64: 1^{\text {cde }}$ \\
\hline $\mathrm{CaCl}_{2}(8.0 \%)$ & $261.8^{\mathrm{cd}}$ & $24.33^{\mathrm{g}}$ & $38.09^{a}$ & $4.20: 1^{\mathrm{bcd}}$ & $6.88: 1^{\mathrm{e}}$ \\
\hline $\mathrm{K}_{2} \mathrm{SO}_{4}(1.0 \%)$ & $272.41^{b c}$ & $33.56^{b}$ & $28.86^{\mathrm{gf}}$ & 4.37: $1^{\mathrm{abcd}}$ & $9.45: 1^{\mathrm{a}}$ \\
\hline $\mathrm{K}_{2} \mathrm{SO}_{4}(1.5 \%)$ & $213.27^{\mathrm{g}}$ & $36.55^{\mathrm{a}}$ & $25.91^{\mathrm{gh}}$ & $3.43: 1^{\mathrm{f}}$ & $8.41: 1^{a b c}$ \\
\hline $\mathrm{K}_{2} \mathrm{SO}_{4}(2 \%)$ & $232.95^{\mathrm{f}}$ & $30.98^{\mathrm{bcd}}$ & $31.45^{\text {def }}$ & $3.74: 1^{\text {ef }}$ & $7.41: 1^{\text {cde }}$ \\
\hline Borax $(1.0 \%)$ & $250.07^{\mathrm{de}}$ & $28.61^{\text {def }}$ & $33.68^{\text {bcd }}$ & 4.03: $1^{\mathrm{cde}}$ & $7.52: 1^{\text {cde }}$ \\
\hline Borax (1.5\%) & $262.86^{\mathrm{cd}}$ & $25.55^{\mathrm{fg}}$ & $36.87^{\mathrm{ab}}$ & $4.21: 1^{\mathrm{bcd}}$ & $7.13: 1^{\mathrm{de}}$ \\
\hline Borax $(2.0 \%)$ & $290.60^{a}$ & $29.93^{\mathrm{cd}}$ & $32.53^{\text {ed }}$ & 4.68: $1^{\mathrm{a}}$ & $8.96: 1^{\mathrm{ab}}$ \\
\hline $\mathrm{ZnSO}_{4}(0.2 \%)$ & $245.24^{\text {eff }}$ & $32.67^{b c}$ & $29.75^{\mathrm{ef}}$ & $3.93: 1^{\mathrm{de}}$ & $8.25: 1^{\mathrm{abcd}}$ \\
\hline $\mathrm{ZnSO}_{4}(0.4 \%)$ & $248.91^{\mathrm{et}}$ & $30.56^{\mathrm{bcd}}$ & $31.86^{\text {det }}$ & $3.98: 1^{\text {cde }}$ & $7.81: 1^{\text {bcde }}$ \\
\hline $\mathrm{ZnSO}_{4}(0.8 \%)$ & $275.55^{b c}$ & $29.12^{\mathrm{ed}}$ & $33.33^{\mathrm{cd}}$ & $4.41: 1^{\mathrm{abc}}$ & $8.27: 1^{\text {abcd }}$ \\
\hline Control & $213.66^{\mathrm{g}}$ & $37.13^{\mathrm{a}}$ & $25.29^{h}$ & $3.43: 1^{f}$ & $8.46: 1^{\mathrm{abc}}$ \\
\hline
\end{tabular}

Value indicates mean of three replicates. Different letters in the same column indicate significant differences at $\mathrm{P} \leq 0.05$ (Duncan's Multiple Range Test)

Table.4 Effect of chemicals on pulp, peel and stone proportion of mango cv. Langra

\begin{tabular}{|c|c|c|c|}
\hline Treatments & Pulp (\%) & Peel (\%) & Stone (\%) \\
\hline $\mathrm{CaCl}_{2}(4.0 \%)$ & $82.25^{\mathrm{a}}$ & $6.65^{\mathrm{g}}$ & $11.09^{a b c}$ \\
\hline $\mathrm{CaCl}_{2}(6.0 \%)$ & $81.62^{\mathrm{ab}}$ & $7.69^{\mathrm{efg}}$ & $10.69^{\mathrm{abcd}}$ \\
\hline $\mathrm{CaCl}_{2}(8.0 \%)$ & $80.75^{\mathrm{ab}}$ & $7.50^{\mathrm{gf}}$ & $11.75^{\mathrm{a}}$ \\
\hline $\mathrm{K}_{2} \mathrm{SO}_{4}(1.0 \%)$ & $81.36^{\mathrm{ab}}$ & $10.02^{b c}$ & $8.61^{\mathrm{g}}$ \\
\hline $\mathrm{K}_{2} \mathrm{SO}_{4}(1.5 \%)$ & $77.34^{\mathrm{d}}$ & $13.26^{\mathrm{a}}$ & $9.40^{\text {efg }}$ \\
\hline $\mathrm{K}_{2} \mathrm{SO}_{4}(2 \%)$ & $78.87^{\mathrm{cd}}$ & $10.48^{\mathrm{a}}$ & $10.64^{\text {abcde }}$ \\
\hline Borax (1.0\%) & $80.05^{b c}$ & $9.16^{\mathrm{cd}}$ & $10.78^{\mathrm{abcd}}$ \\
\hline Borax $(1.5 \%)$ & $80.81^{a b}$ & $7.86^{\mathrm{et}}$ & $11.33^{\mathrm{ab}}$ \\
\hline Borax $(2.0 \%)$ & $82.30^{\mathrm{a}}$ & $8.48^{\text {def }}$ & $9.216^{\mathrm{fg}}$ \\
\hline $\mathrm{ZnSO}_{4}(0.2 \%)$ & $79.71^{b c}$ & $10.62^{b}$ & $9.668^{\operatorname{defg}}$ \\
\hline $\mathrm{ZnSO}_{4}(0.4 \%)$ & $79.91^{b c}$ & $9.83^{b c}$ & $10.25^{\text {bcdef }}$ \\
\hline $\mathrm{ZnSO}_{4}(0.8 \%)$ & $81.51^{\mathrm{ab}}$ & $8.62^{\mathrm{de}}$ & $9.87^{\text {cdefg }}$ \\
\hline Control & $77.35^{\mathrm{d}}$ & $13.47^{\mathrm{a}}$ & $9.18^{\mathrm{gf}}$ \\
\hline
\end{tabular}

Value indicates mean of three replicates. Different letters in the same column indicate significant differences at $\mathrm{P} \leq 0.05$ (Duncan's Multiple Range Test) 
Table.5 Effect of chemicals on TSS, titratable acidity and sugar content of mango cv. Langra

\begin{tabular}{|c|c|c|c|c|}
\hline Treatments & $\operatorname{TSS}\left({ }^{0} \mathrm{~B}\right)$ & $\begin{array}{c}\text { Titratable } \\
\text { Acidity }(\%)\end{array}$ & $\begin{array}{c}\text { TSS:TA } \\
\text { ratio }\end{array}$ & $\begin{array}{l}\text { Total sugars } \\
\text { content }(\%)\end{array}$ \\
\hline $\mathrm{CaCl}_{2}(4.0 \%)$ & $20.64^{a}$ & $0.27^{\mathrm{bc}}$ & $76.45: 1^{c}$ & $11.93^{\mathrm{a}}$ \\
\hline $\mathrm{CaCl}_{2}(6.0 \%)$ & $19.98^{\mathrm{b}}$ & $0.26^{\mathrm{cd}}$ & $76.97: 1^{c}$ & $11.08^{b}$ \\
\hline $\mathrm{CaCl}_{2}(8.0 \%)$ & $19.84^{b c}$ & $0.26^{\mathrm{cd}}$ & $76.37: 1^{c}$ & $10.47^{\mathrm{bc}}$ \\
\hline $\mathrm{K}_{2} \mathrm{SO}_{4}(1.0 \%)$ & $20.02^{b}$ & $0.28^{\mathrm{ab}}$ & $71.53: 1^{d}$ & $7.65^{\mathrm{e}}$ \\
\hline $\mathrm{K}_{2} \mathrm{SO}_{4}(1.5 \%)$ & $20.18^{b}$ & $0.26^{\mathrm{cd}}$ & $77.68: 1^{c}$ & $7.31^{\text {ef }}$ \\
\hline $\mathrm{K}_{2} \mathrm{SO}_{4}(2 \%)$ & $19.78^{b c}$ & $0.28^{\mathrm{ab}}$ & $70.67: 1^{d}$ & $7.19^{\text {ef }}$ \\
\hline Borax $(1.0 \%)$ & $21.08^{\mathrm{a}}$ & $0.25^{\mathrm{de}}$ & $84.39: 1^{\mathrm{a}}$ & $12.04^{\mathrm{a}}$ \\
\hline Borax $(1.5 \%)$ & $20.87^{\mathrm{a}}$ & $0.26^{\mathrm{cd}}$ & $80.31: 1^{a b c}$ & $11.87^{\mathrm{a}}$ \\
\hline Borax $(2.0 \%)$ & $20.16^{b}$ & $0.26^{\mathrm{cd}}$ & $77.59: 1^{\mathrm{c}}$ & $11.00^{\mathrm{b}}$ \\
\hline $\mathrm{ZnSO}_{4}(0.2 \%)$ & $19.06^{\text {ed }}$ & $0.24^{\mathrm{e}}$ & $79.50: 1^{\mathrm{abc}}$ & $9.47^{\mathrm{d}}$ \\
\hline $\mathrm{ZnSO}_{4}(0.4 \%)$ & $19.46^{\mathrm{cd}}$ & $0.25^{\mathrm{de}}$ & $77.91: 1^{\mathrm{bc}}$ & $9.48^{\mathrm{d}}$ \\
\hline $\mathrm{ZnSO}_{4}(\mathbf{0 . 8 \%})$ & $19.88^{b c}$ & $0.24^{\mathrm{e}}$ & $82.87: 1^{\mathrm{ab}}$ & $10.11^{\mathrm{c}}$ \\
\hline Control & $18.98^{\mathrm{e}}$ & $0.29^{\mathrm{a}}$ & $65.47: 1^{\mathrm{e}}$ & $6.85^{\mathrm{f}}$ \\
\hline
\end{tabular}

Value indicates mean of three replicates. Different letters in the same column indicate significant differences at $\mathrm{P} \leq 0.05$ (Duncan's Multiple Range Test)

Table.6 Effect of chemicals on, carotenoids, ascorbic acid, phenolics content and antioxidant activity of mango cv. Langra

\begin{tabular}{|c|c|c|c|c|}
\hline Treatments & $\begin{array}{c}\text { Total } \\
\text { carotenoids } \\
\text { (mg/100 gm } \\
\text { FW) }\end{array}$ & $\begin{array}{c}\text { Ascorbic acid } \\
(\mathbf{m g} / 100 g m \text { FW) }\end{array}$ & $\begin{array}{c}\text { Total } \\
\text { phenolics(mg } \\
\text { GAE/g FW) }\end{array}$ & $\begin{array}{c}\text { Antioxidant } \\
\text { activity } \\
\text { ( } \begin{array}{c}\text { mol TE/g } \\
\text { FW) }\end{array}\end{array}$ \\
\hline $\mathrm{CaCl}_{2}(4.0 \%)$ & $1.56^{\mathrm{c}}$ & $72.33^{\mathrm{ab}}$ & $6.20^{\mathrm{ab}}$ & $4.68^{\mathrm{a}}$ \\
\hline $\mathrm{CaCl}_{2}(6.0 \%)$ & $1.44^{\mathrm{g}}$ & $70.12^{b c}$ & $5.90^{\mathrm{abc}}$ & $4.26^{\mathrm{a}}$ \\
\hline $\mathrm{CaCl}_{2}(8.0 \%)$ & $1.46^{\mathrm{f}}$ & $68.44^{\mathrm{cd}}$ & $5.70^{\mathrm{bcd}}$ & $4.74^{\mathrm{a}}$ \\
\hline $\mathrm{K}_{2} \mathrm{SO}_{4}(1.0 \%)$ & $1.52^{\mathrm{d}}$ & $64.23^{\mathrm{e}}$ & $5.60^{\text {bcde }}$ & $4.71^{\mathrm{a}}$ \\
\hline $\mathrm{K}_{2} \mathrm{SO}_{4}(1.5 \%)$ & $1.50^{\mathrm{e}}$ & $64.67^{\mathrm{e}}$ & $4.93^{\mathrm{fg}}$ & $4.51^{\mathrm{a}}$ \\
\hline $\mathrm{K}_{2} \mathrm{SO}_{4}(2 \%)$ & $1.42^{\mathrm{h}}$ & $62.25^{\text {ef }}$ & $5.23^{\text {defg }}$ & $4.71^{\mathrm{a}}$ \\
\hline Borax $(1.0 \%)$ & $1.64^{\mathrm{a}}$ & $75.26^{\mathrm{a}}$ & $6.34^{\mathrm{a}}$ & $5.16^{\mathrm{a}}$ \\
\hline Borax (1.5\%) & $1.58^{b}$ & $68.67^{c}$ & $5.60^{\text {bcde }}$ & $5.03^{\mathrm{a}}$ \\
\hline Borax $(2.0 \%)$ & $1.55^{c}$ & $73.33^{\mathrm{ab}}$ & $5.50^{\text {cdef }}$ & $4.98^{a}$ \\
\hline $\mathrm{ZnSO}_{4}(0.2 \%)$ & $1.58^{b}$ & $63.98^{\mathrm{e}}$ & $4.90^{\mathrm{fg}}$ & $5.10^{\mathrm{a}}$ \\
\hline $\mathrm{ZnSO}_{4}(0.4 \%)$ & $1.52^{d}$ & $64.33^{e}$ & $5.20^{\operatorname{detg}}$ & $4.98^{\mathrm{a}}$ \\
\hline $\mathrm{ZnSO}_{4}(0.8 \%)$ & $1.46^{\mathrm{f}}$ & $65.28^{\mathrm{de}}$ & $5.00^{\text {efg }}$ & $4.68^{a}$ \\
\hline Control & $1.41^{\mathrm{h}}$ & $60.22^{t}$ & $4.60^{g}$ & $4.03^{\mathrm{a}}$ \\
\hline
\end{tabular}

Value indicates mean of three replicates. Different letters in the same column indicate significant differences at $\mathrm{P} \leq 0.05$ (Duncan's Multiple Range Test) 


\section{Total sugars}

Significant variation was recorded among various treatments with respect to sugar content and clearly revealed in table-5. Total sugar gradually increased in all the treatment than that of control. The maximum total sugar content $(12.04 \%)$ was recorded in borax $1 \%$ followed by $\mathrm{CaCl}_{2} 4 \%$ while minimum $(6.85 \%)$ was found in control.

Boron facilitates sugar transport within plants and reacts with sugar to form a sugar borate complex which reduces its consumption during metabolic processes (Gauch and Dugger, 1953; Stamper et al., 1999; Rajput and Chand, 1976). These results were supported by results obtained earlier by Nehete et al., 2011; Bhatt et al., 2012; Gaur et al., 2014.

\section{Total carotenoids}

The data illustrated in table- 6 showed that all the treatments significantly influenced total carotenoids content. Highest total carotenoids was observed in borax $1 \%(1.64 \mathrm{mg} / 100 \mathrm{gm})$ followed by borax $1.5 \%$ whereas the least total carotenoids was recorded in control $(1.41 \mathrm{mg} / 100 \mathrm{gm}$ FW).

\section{Ascorbic acid content}

Foliar spray of borax $1 \%$ showed significantly highest ascorbic acid (75.26 mg/100gm) followed by borax $2 \%$ while the minimum ascorbic acid $(60.20 \mathrm{mg} / 100 \mathrm{gm})$ was found in control (Table-6).

Higher level of ascorbic acid by application of boron might be due to higher content of ascorbic acid as synthesized from sugar. Similar effect was observed by Chandra et al., 1994; Gaur et al., 2014; Bhatt et al., 2012; Bhowmick and Banik, 2011; Nehete et al., 2011.

\section{Total phenolic content}

Highest total phenolic content $(6.34 \mathrm{mg}$ $\mathrm{GAE} / 100 \mathrm{~g} \mathrm{FW}$ ) was observed in borax $1 \%$ followed by $\mathrm{CaCl}_{2} 4 \%$ whereas it was lowest in control (4.60 mg GAE/100g FW). High boron concentration resulted in the increase of gene expression and enzyme activities involved in phenolic biosynthesis (Song et al., 2015). The result was accordance with earlier reports of Herrera-Rodriguez et al., 2010 and Davarpanah et al., 2015.

\section{Total antioxidant activity}

Antioxidant activity increases in all treatments with no significant differences between treated and untreated fruit. However, the data shown in table- 6 indicated that among treatments, borax $1 \%$ had comparatively highest antioxidant activity (5.16 $\mu$ mol Trolox equivalent/100g FW). Similar results were found in blue berry and pomegranate (Merino-Gergichevich et al., 2016; Davarpanah et al., 2015).

On the basis of results summarized above, it can be concluded that foliar application of calcium, potassium, boron and zinc showed significant variation in yield, fruit development, and its quality attributes of the mango cv. Langra.

Present investigation concluded that borax @ $1 \%$ and $\mathrm{CaCl}_{2} @ 4 \%$ was most efficient treatment for enhancing yield and retaining the quality of mango fruits.

\section{Acknowledgements}

The authors are grateful to the Chairman, Department of Fruit and Fruit Technology, Bihar Agricultural University, Sabour, Bhagalpur for providing necessary facilities and financial support for research work. 


\section{References}

A.O.A.C. 2000. Official Methods of Analysis. $17^{\text {th }}$ ed. Association of Official Analytical Chemists, Gaithersburg, MD.

Anonymous. 2015. Indian Horticulture Database (Chief ed. Gandhi C.P.). National Horticulture Board, Ministry of Agriculture, Govt. of India, Gurgaon, Haryana, India.

Apak, R., Guclu, K., Ozyurek, M., Karademir, S. E. 2004. Novel total antioxidant capacity index for dietary polyphenol and vitamins $\mathrm{C}$ and $\mathrm{E}$, using their cupric ion reducing capability in the presence of neocuproine, CUPRAC method. Journal of Agriculture and Food Chemistry. 52:7970-7981.

Baiea M. H. M., El-Sharony T. F., Eman A. A. and El- Moneim, A. 2015. Effect of different forms of potassium on growth, yield and fruit quality of mango cv. Hindi. International Journal of Chem Tech Research. 8(4), 1581-1587.

Bains, K. S., Bajwa, G. S. and Singh, Z. 1997. Abscission of mango fruitlets in relation to endogenous concentrations of IAA, $\mathrm{GA}$ and $\mathrm{ABA}$ in pedicels and fruitlets. Fruits. 52: 159-165.

Bhatt, A., Mishra, N. K., Mishra, D. K. and Singh, C. P. 2012. Foliar application of potassium, calcium, zinc and boron enhanced yield, quality and shelf life of mango. Horticultural Flora Research Spectrum. 1(4): 300-305.

Bhowmick, N. and Banik, B. C. 2011. Influence of pre-harvest foliar application of growth regulators and micronutrients on mango cv. Himsagar. Indian Journal of Horticulture. 68(1): 103-107.

Bhowmick, N., Banik, B. C., Hasan, M. A. and Ghosh, B. 2012. Response of preharvest foliar application of zinc and boron on mango cv. Amrapali under New Alluvial Zone of West Bengal. Indian Journal of Horticulture. 69(3): 428-431.
Bhusan, L. P. and Panda, C. 2015. Effect of pre-harvest chemical treatments and mulching on quality of mango, Mangifera indica L cv. Amrapali. International Journal of Farm Sciences. 5(4): 132-138.

Blevins, D. G. and Lukaszewski, K. M. 1998. Boron in Plant Structure and Function. Annual Review of Plant Physiology and Plant Molecular Biology. 49:481-500.

Cakmak, I. 2005. The role of potassium in alleviating detrimental effects of abiotic stresses in plants. Journal of Plant Nutrition and Soil Science. 168: 521-530.

Chandra, R, Govind, S. and Basuchaudhari, P. 1994. Preharvest sprays of calcium nitrate and alar on quality and postharvest behaviour of guava fruits. Indian Journal of Hill Farming. 7(1): 51-56.

Davarpanah, S., Tehranifar, A. Davarynejad, G., Abadia, J. and Khorasani, R. 2016. Effects of foliar applications of zinc and boron nano-fertilizers on pomegranate (Punica granatum cv. Ardestani) fruit yield and quality. Scientia Horticulturae. 210:1-8.

Dutta, P. and Dhua, R. S. 2002. Improvement on fruit quality of Himsagar mango through application of zinc, iron and manganese. The Horticulture Journal. 15 (2): 1-9.

Gauch, H. G. and Duggar, W. M. 1953. The role of boron in the translocation of sucrose. Plant Physiology. 28: 457-66.

Gaur, B., Beer, K., Hada, T. S., Kanth, N. and Syamal, M. M. 2014. Studies on the effect of foliar application of nutrients and $\mathrm{GA}_{3}$ on fruit yield and quality of winter season guava. The Ecoscan. 6: 479-483.

Herrera-Rodriguez, M. B. A. GonzalezFontes, R. J., Camacho-Cristobal, J. J., Maldonado, J. M. and Navarro-Gochicoa, M. T. 2010. Role of boron in vascular plants and response mechanisms to boron stresses. Plant Stress. 4 (2):115-122. 
Jones, E. and Hughes, R. E. 1983. Foliar ascorbic acid in some angiosperms. Phytochemistry. 22: 2493-2499.

Karemera, N. J. U. and Habimana, S. 2014. Effect of $\mathrm{CaCl}_{2}$ sprays on ripening, shelflife and physical chemical proprieties of mango fruits (Mangifera indica L.) cv. Totapuri. Herald Journal of Agriculture and Food Science Research. 3(2): 61-66.

Kavitha, M., Kumar, N. and Jeyakumar, P. 2000. Role of Zinc and Boron on fruit yield and associated characters in papaya cv. Co5. South Indian Horticulture. 48(6): 6-10.

Kulkarni, S. S. and Yewale, P. H. 2012. Effect of mulching and chemicals for improving yield and quality of mango cv. Keshar. International Journal of Forestry and Crop Improvement. 3(2):137-139.

Kumar, A. R., Kumar, N. and Kavino, M. 2006. Role of potassium in fruit crops A review. Agriculture Review. 27(4): 284-291.

Lane, J. H. and Eynon, L. 1923. Determination of reducing sugars by means of Fehling's solution with methylene blue as internal indicator. Journal of the Chemical Society Trans. 32-36.

Meena, D., Tiwari, D. R. and Singh, O. P. 2014. Effect of nutrient spray on growth, fruit yield and quality of aonla. Annals of Plant and Soil Research. 16(3): 242-245.

Merino-Gergichevich, C., Pacheco, E. and Reyes-Diaz, M. 2016. The effect of foliar boron spraying on the fruit features of Brigitta and Legacy high bush blueberry (Vaccinium corymbosum) cultivars. Cienciae Investigacion Agraria. 43(3):452-463.

Nehete, D. S., Padhiar, B. V., Shah, N. I., Bhalero, P. P., Kolambe, B. N. and Bhalero, R. R. 2011. Influence of micronutrient spray on flowering, yield, quality and nutrient in leaf of mango cv. Kesar. The Asian Journal of
Horticulture. 6(1): 63-67.

Pandey, S. K. and Jain, P. K. 2014. Effect of micronutrients, growth regulators and bio-fertilizers on biochemical composition of mango cv. Langra. International Journal of Scientific Research. 3(12): 146-147.

Pathak, M., Bauri, F. K., Misra, D. K., Bandyopadhyay, B. and Chakraborty, K. 2011. Application of micronutrients on growth, yield and quality of banana. Journal Crop and Weed. 7:52-54.

Pettigrew, W. T. 2008. Potassium influences on yield and quality production for maize, wheat, soybean and cotton. Plant Physiology. 133: 670-681.

Raese, J. T. and Drake, S. R. 2000. Effect of calcium sprays, time of harvest, cold storage, and ripeness on fruit quality of 'Anjou' pears. Journal of Plant Nutrition. 23: 843-853.

Rajput, C. B. S. and Chand, S. 1976. Effect of boron and zinc on the physico-chemical composition of guava fruits (Psidium guajava L.). Journal of National Agriculture Science of Ceylon. 13: 49-54.

Rani, R. and Brahmachari, V. S. 2001. Effect of foliar application of calcium, zinc and boron on cracking and physico-chemical composition of litchi. Orissa Journal of Horticulture. 29(1): 50-54.

Roy, S. K. 1973. A simple and rapid method for estimation of total carotenoid pigments in mango. Journal of Food Science Technology. 10:38-42.

Sankar, C., Saraladevi, D. and Parthiban, S. 2013. Influence of pre-harvest foliar application of micronutrients and sorbitol on pollination, fruit set, fruit drop and yield in mango (Mangifera indica L.) cv. Alphanso. Asian Journal of Horticulture. 8(2): 635-640.

Sarolia, D. K, Rathore, N. S. and Rathore, R. S. 2007. Response of $\mathrm{ZnSO}_{4}$ and iron suphate sprays on growth and productivity of guava (Psidium guajava 
L.) cv. Sardar. Current Agriculture. 31(2): 73-77.

Selvaraj, Y., Raja, M. E. and Rawal R. D. 2000. Biochemical studies on breakdown a ripening disorder on mango fruits. Indian Journal of Horticulture, 57:183188.

Singh, A. K., Singh, C. P. and Chauhan, P. 2012. Effect of pre-harvest chemical treatments and mulching on quality and marketability of Dashehari mango. Indian Journal of Horticulture. 69(4): $462-466$.

Singh, A. K., Singh, C. P. and Chauhan, P. 2012. Effect of pre-harvest chemical treatments and mulching on quality and marketability of Dashehari mango. Indian Journal of Horticulture. 69(4): 462-466.

Singh, A. K., Singh, C. P., Lal, S. and Pratibha. 2013. Effect of micronutrients and sorbitol on fruit set, yield and quality of mango cv. Dashehari. Progressive Horticulture. 45 (1): 43-48.

Singh, G and Maurya, A. N. 2004. Effect of micronutrients on bearing of mango cv. Mallika. Progressive Horticulture. 4: 4750.

Singleton, V. L. and Ross, J. A. 1965. Colorimetry of total phenolics with phosphomolybdic-phosphotungstic acid reagents. American Journal of Enology and Viticulture, 16: 144-158.

Song, C. Z., Liu, M. Y., Meng, J. F., Chi, M., Xi, Z. M. and Zhang, Z.W. 2015.
Promoting effect of foliage sprayed zinc sulfate on accumulation of sugar and phenolics in berries of Vitis vinifera $\mathrm{cv}$. merlot growing on zinc deficient soil. Molecules. 20: 2536-2554.

Sourour, M. M. 2000. Effect of foliar application of some micronutrient forms on growth, yield, fruit quality and leaf mineral composition of Valencia orange trees grown in North Sinai. Alexandria Journal of Agriculture Research45(1): 269-285.

Stampar, F., Hudina, M. and Dolence, K. 1999. Influence of foliar fertilizer on yield and quality of apple (Mallus domestica Borkh.). Kumar Academic Publisher. Pp. 91-94.

Taha, R. A., Hassan, H. S. A. and Shaaban, E.A. 2014. Effect of Different Potassium Fertilizer Forms on Yield, Fruit Quality and Leaf Mineral Content of Zebda Mango Trees. Middle-East Journal of Scientific Research. 21 (3): 518-524.

Yadav, V. Singh, P. N. and Yadav, P. 2013. Effect of foliar fertilization of boron, zinc, and iron on fruit growth and yield of low-chilled peach cv. Sharbati. International Journal of Scientific and Research Publications. 3 (8):2250-3153.

Zaman, Q. and Achumann, A. W. 2006. Nutrient management zones of citrus based variation in soil properties and tree performance. Precision Agriculture. 7(1): 45-63.

\section{How to cite this article:}

Anugya Kumari, Rewati Raman Singh and Manoj Kundu. 2020. Effect of Pre-harvest Application of $\mathrm{Ca}, \mathrm{K}, \mathrm{B}$ and $\mathrm{Zn}$ on Yield and Quality of Mango (Mangifera indica L.) cv. Langra. Int.J.Curr.Microbiol.App.Sci. 9(06): 892-902. doi: https://doi.org/10.20546/ijcmas.2020.906.112 